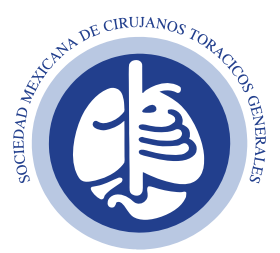

Vol. 1, Núm. 1

Enero-Abril 2020 pp 4-9

cirujanos torácicos

generales, fundación.

Keywords: Society, general thoracic surgeons, foundation.
* Cirujano Torácico General, adscrito al Servicio de Cirugía, Hospital General de Tijuana, ISESALUD;

"Cirujano Torácico General, Sanatorio Fátima de Córdoba, Veracruz.

\footnotetext{
₹ Presentado en el VII Congreso Internacional de la Sociedad Mexicana de Cirujanos Torácicos Generales, Monterrey, Nuevo León, Septiembre 2018.
}

Recibido: 13/12/2018

Aceptado: 13/12/2018

Correspondencia: Carlos A Olivares-Torres

E-mail: caolivarest@gmail.com
REVISTA

MEXICANA DE

CIRUGÍA

TORÁCICA

GENERAL

doi: $10.35366 / 94446$ https://dx.doi.org/10.35366/94446

\author{
Perspectivas
}

\title{
De la Sociedad hacia el Colegio ${ }^{\ddagger}$
}

\author{
From Society to the College \\ Carlos A Olivares-Torres, ${ }^{*}$ José Antonio Aburto-Salomón"
}

\begin{abstract}
RESUMEN
La Sociedad Mexicana de Cirujanos Torácicos Generales tiene sus inicios en los albores del siglo XXI, formando una nueva sociedad a partir de 2010, tomando forma jurídica en la Hacienda de los Morales de la Ciudad de México en mayo del mismo año. A la fecha ha organizado siete congresos llevados a cabo en diferentes puntos del país con cambios en la presidencia en forma ordenada, con la firme idea de la formación del Colegio de Cirujanos Torácicos, innovando y realizando los procedimientos que los pacientes requieren y de esa manera engrandecer más la especialidad.
\end{abstract}

\section{ABSTRACT}

The Mexican Society of General Thoracic Surgeons has its beginnings at the dawn of the 21st century, forming a new society as of 2010, taking legal form at the Hacienda de los Morales in Mexico City in May of the same year, to date with the holding of VII congresses held in different parts of the country with changes in the presidency in an orderly manner, with the firm idea of forming the College of Thoracic Surgeons, innovating and performing the procedures that patients require and thus further enhance the specialty.

\section{INTRODUCCIÓN}

Los cirujanos de tórax, o más bien los cirujanos torácicos generales, siempre han buscado una identidad propia con esa inquietud que caracteriza a todo cirujano de siempre indagar más y no quedarse estático; los miembros de nuestra sociedad no son la excepción. Después de pertenecer y trabajar con otras sociedades afines se decidió tener nuestra propia identidad y formar la Sociedad Mexicana de Cirujanos Torácicos Generales, una historia que se cuenta aparte.

La Sociedad Mexicana de Cirujanos Torácicos Generales de México, mejor conocida como SMCTG, tiene su inicio en los albores del presente siglo XXI al tener los cirujanos torácicos del país una identidad y un nombre propios, mismos que la Sociedad Mexicana de Neumología y Cirugía de Tórax no podía cumplir debido a que los neumólogos de esas sociedades tienen una mayoría abrumadora y no entienden los problemas y las necesidades de los cirujanos ni las complejidades de la cirugía de tórax.

Fue en la Ciudad de México, en específico en la Hacienda de los Morales en noviembre de 2004, cuando reunidos los doctores Aburto, Avilez, Castillo, Méndez, Olivares, Rivero

Citar como: Olivares-Torres CA, Aburto-Salomón JA. De la Sociedad hacia el Colegio. Rev Mex Cir Torac Gen. 2020; 1(1):4-9 


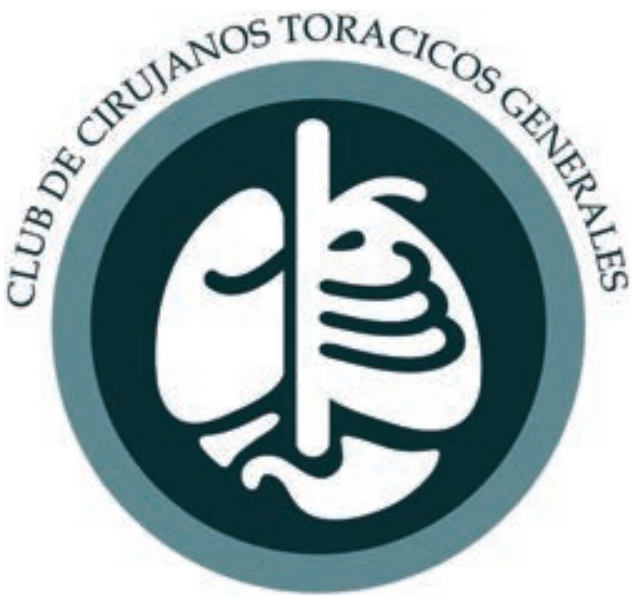

Figura 1: El logo muestra los aspectos de nuestra especialidad como cirugía de vía aérea, pulmonar, diafragmática, pared costal y tubo digestivo alto.

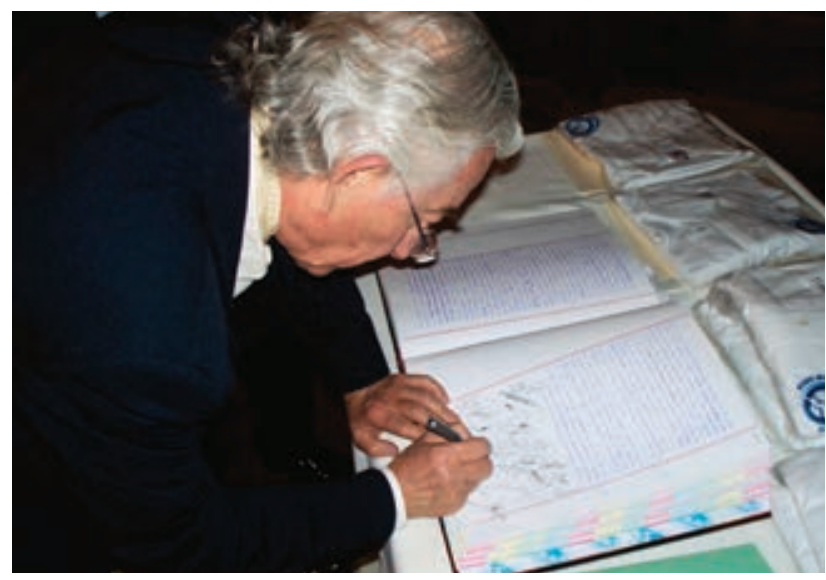

Figura 2: El Dr. Carlos Ibarra Pérez, socio fundador, firmando el acta constitutiva en la Hacienda de los Morales, Ciudad de México, mayo de 2010. y Schcolnik se planteó tener una sociedad aparte de la SMNYCT para disponer de foros de discusión, página de Internet, consensos en cirugía de tórax, trabajar conjuntamente con The Society of Thoracic Surgeons y el General Thoracic Surgical Club, y promover la especialidad sólo a través de cirujanos.

Para esto se compró un dominio, una página de Internet y se mandó diseñar un logo.

Se nombró una mesa directiva, quedando como presidente el Dr. Graciano Castillo Ortega, se presentaron trabajos y sesiones en conjunto con la SMNYCT y otras sociedades afines con presencia de cirujanos torácicos distinguidos a nivel internacional.

Pero después de una relación tensa con la SMNYCT, se decidió en la ciudad de Guadalajara separarse de dicha sociedad y formar la nuestra, Ilamada Sociedad Mexicana de Cirujanos Torácicos Generales o SMCTG, adoptar el logo propuesto en 2004 como el oficial, y nuevamente se planteó la reunión en mayo de 2010 en la Hacienda de los Morales en la Ciudad de México para firmar el acta constitutiva, cuyo trámite notarial se hizo en Córdoba, Veracruz, y de esta manera formalizar la sociedad, quedando la Mesa Directiva:

Presidente: Dr. Graciano Castillo Ortega Vicepresidente: Dr. Carlos A Olivares Torres Secretario: Dr. José Antonio Aburto Salomón Tesorero: Dr. Jorge Alberto Silva Vivas

Quedó establecido que el término de la presidencia será de dos años y nuestro primer presidente convocó a la primera reunión en la Ciudad de México en septiembre de 2012 con la presencia de

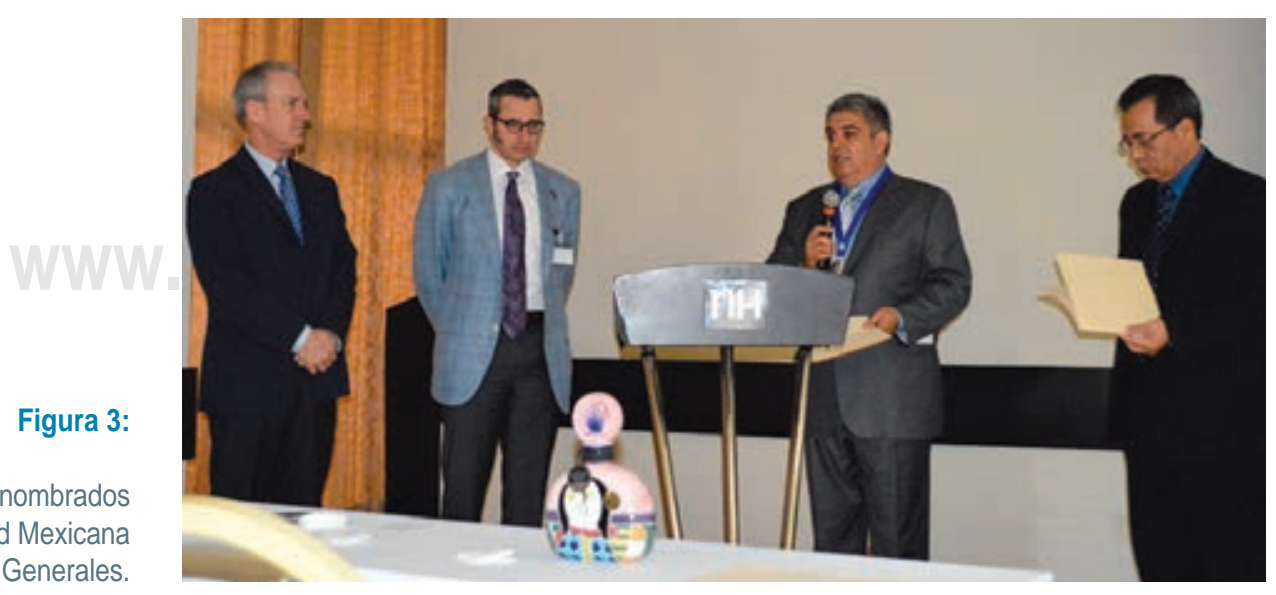

Doug Wood y Rafael Andrade, nombrados miembros honorarios de la Sociedad Mexicana de Cirujanos Torácicos Generales. 
dos profesores internacionales: Doug Wood y Rafael Andrade, los cuales fueron nombrados miembros honorarios.

Se realizó el II Congreso en 2013 de nuevo en la Ciudad de México, el Dr. Graciano Castillo pasó la estafeta al Dr. Carlos A Olivares Torres, asumiendo su rol de segundo presidente de nuestra naciente sociedad y el Dr. Jorge Silva Vivas la vicepresidencia.
Se organizó el III Congreso en la Ciudad de México ya con cambio de rumbo, con un registro más profesional, más casas comerciales y más personal, lo que nos habla de un crecimiento en forma horizontal de la SMCTG con mayor asistencia.

De esta manera se llevó a cabo el III Congreso y se tomó la decisión de sacar el evento de la Ciudad de México, realizando el IV Congreso Internacional

Figura 4:

El Dr. Carlos A Olivares Torres asumiendo su rol de segundo presidente de nuestra naciente sociedad, asumiendo el Dr. Jorge Silva Vivas la vicepresidencia.

III Congreso en la CDMX ya con cambio de rumbo, con un registro más profesional, más casas comerciales y más personal, lo que nos habla de un crecimiento en forma horizontal de la SMCTG con mayor asistencia.
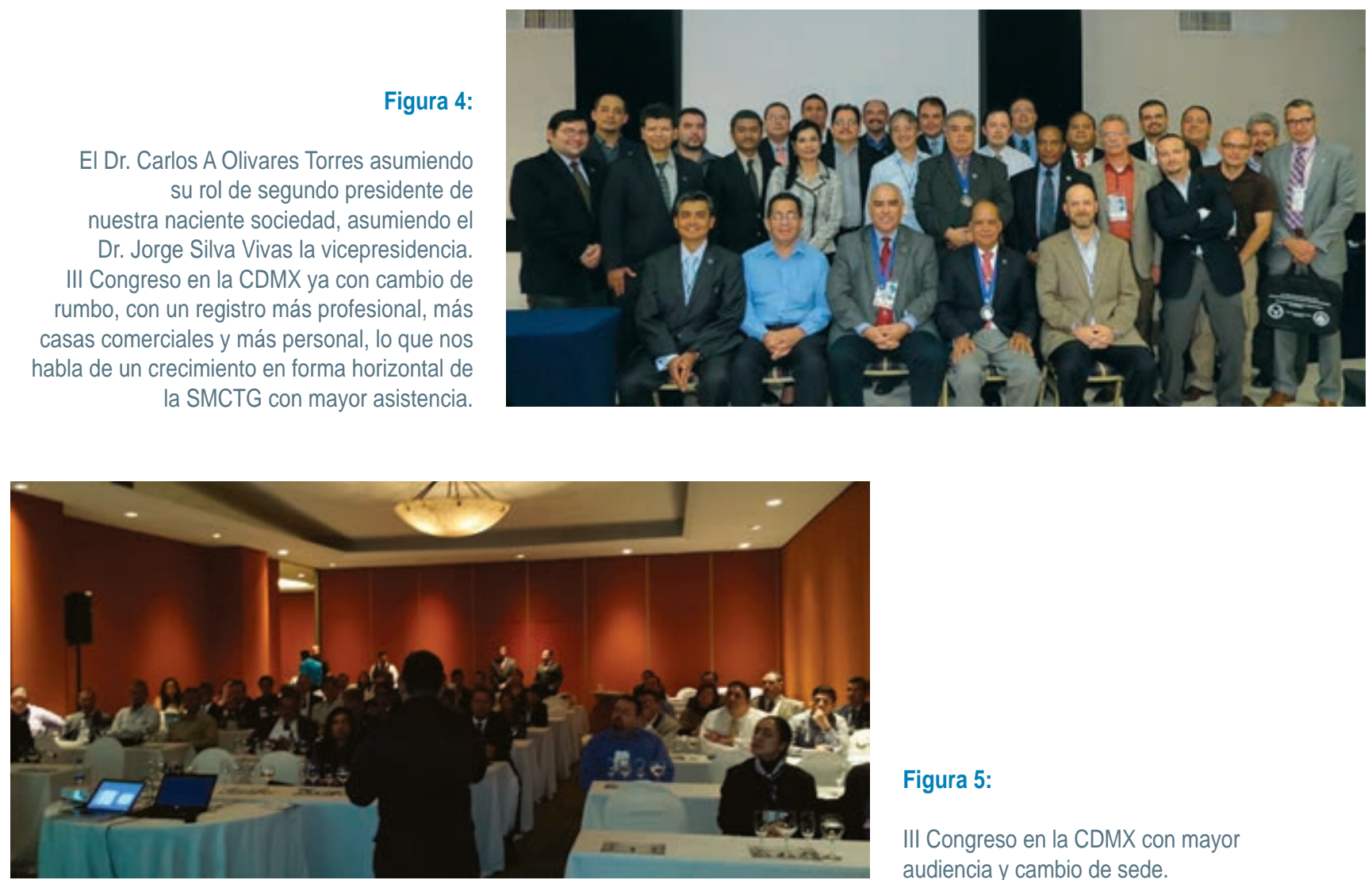

Figura 5:

III Congreso en la CDMX con mayor audiencia y cambio de sede.

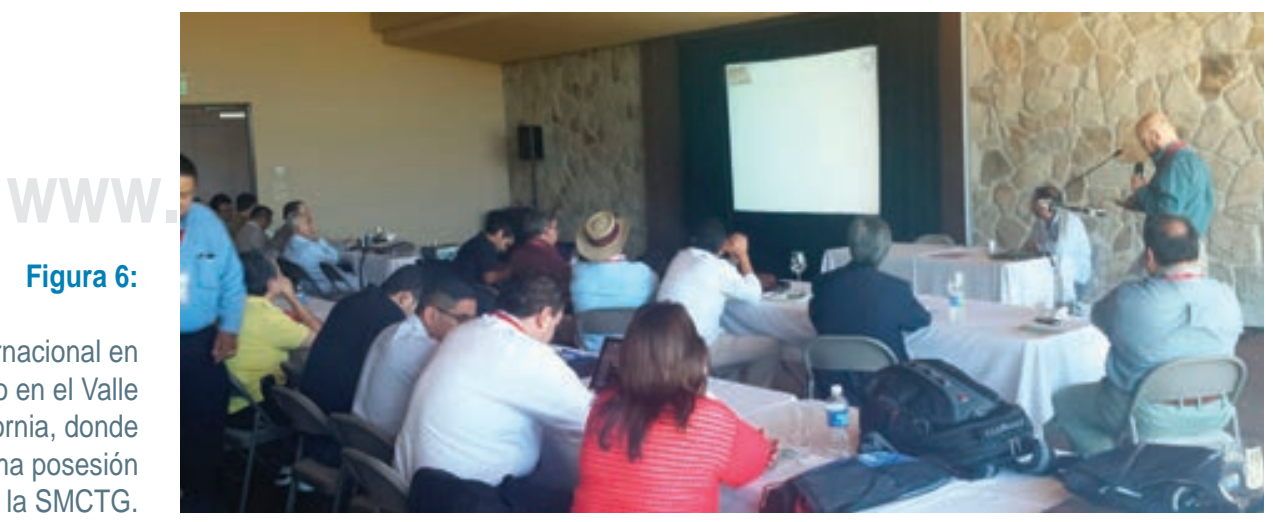




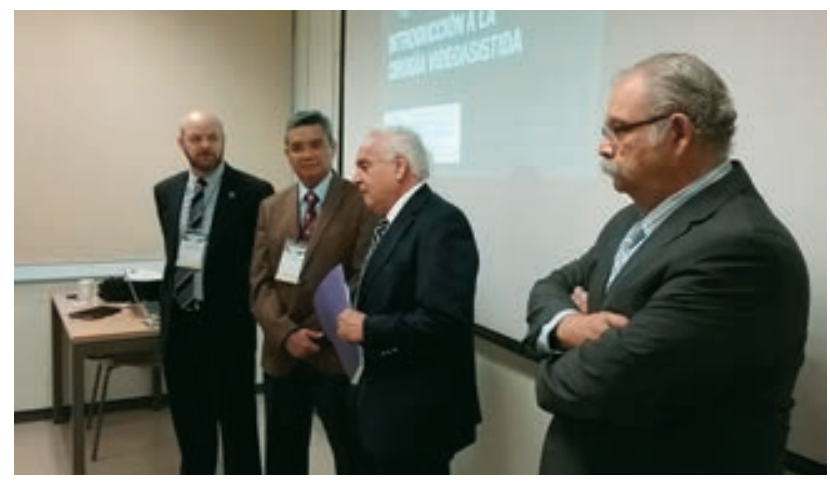

Figura 7: V Congreso: presidente Jorge Silva en la Universidad Nacional Autónoma de México (UNAM) en su Unidad de Posgrado bajo la tutela del Dr. Enrique Guzmán de Alba.

en el Museo de la Vid y el Vino en el Valle de Guadalupe de Baja California, donde el Dr. Jorge Silva Vivas tomó posesión como el tercer presidente de la sociedad.

El V Congreso se celebró una vez más en la Ciudad de México bajo la tutela de nuestro tercer presidente, el Dr. Jorge Silva, iniciando con los cursos de Posgrado en Invasión Mínima en nuestra máxima casa de estudios, la Universidad Nacional Autónoma de México (UNAM) en su Unidad de Posgrado bajo la tutela del Dr. Enrique Guzmán de Alba, además del Posgrado de Endoscopia Intervencionista.

Desafortunadamente, el congreso fue una reunión que se vio empañada por el fallecimiento de uno de nuestros socios fundadores, el Dr. Carlos Montero Cantú; de uno de los iniciadores del Servicio de Cirugía de Tórax del Instituto Nacional de Enfermedades Respiratorias, el Dr. Jesús Rodríguez Esparza; y de uno de los pilares de la cirugía torácica a nivel mundial, el Dr. F Griffith Pearson. Se rindieron sendos homenajes.

EI VI Congreso programado en septiembre de 2017 se llevó a cabo a pesar de los hechos ocurridos tras el temblor de gran magnitud del 19 de septiembre; de esta manera los cirujanos de tórax mostraron su solidaridad con la Ciudad de México. En esta ocasión, el Dr. José Antonio Aburto asumió la cuarta presidencia de nuestra sociedad y fueron elegidos el Dr. Ulises Loyola y el Dr. Marco Antonio Iñiguez como vicepresidente y secretario, respectivamente.

En su toma de protesta, el Dr. Aburto anunció la formación del Colegio de Cirujanos Torácicos con el objetivo de fungir como la rama ejecutiva de la fun-
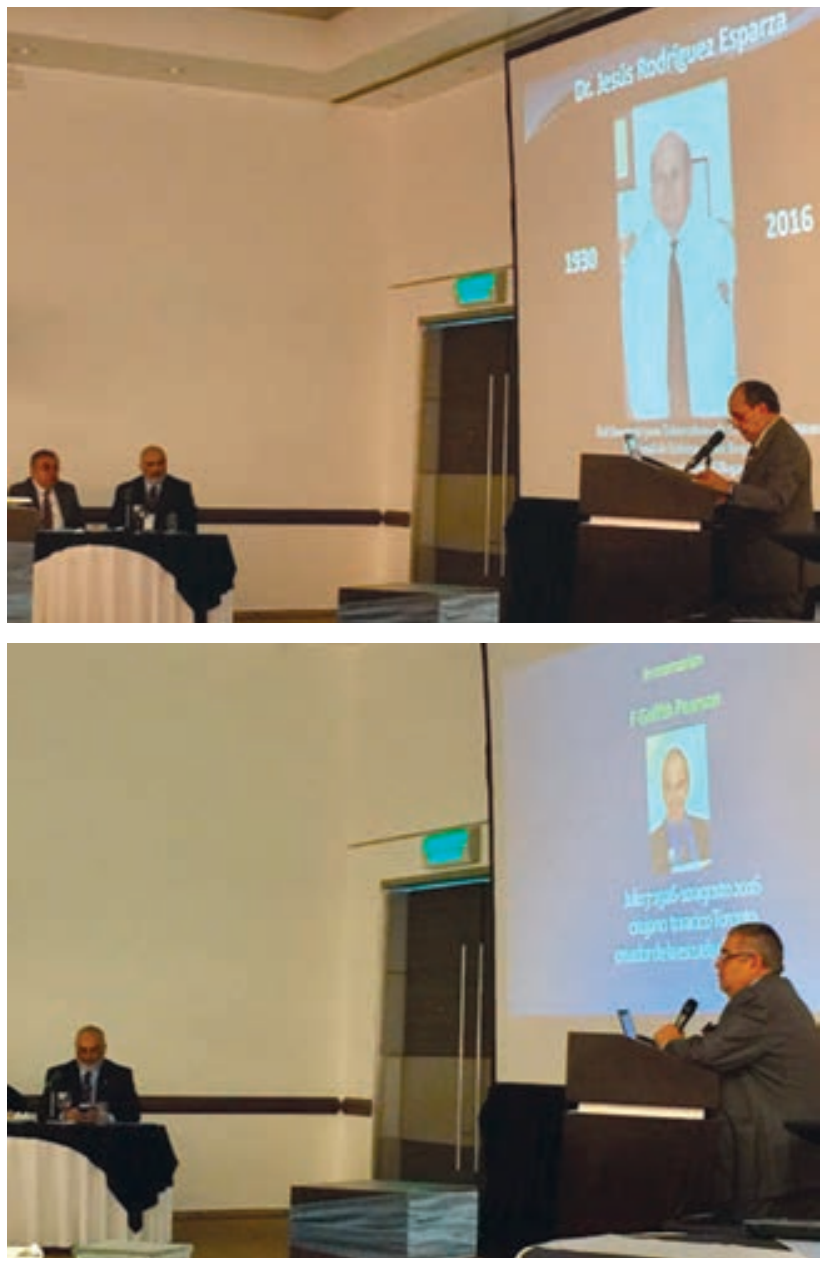

Figuras 8 y 9: Homenaje al ex-jefe del Servicio de Cirugía de Tórax del Instituto Nacional de Enfermedades Respiratorias, el Dr. Jesús Rodríguez Esparza, y a uno de los pilares de la cirugía torácica a nivel mundial, el Dr. F Griffith Pearson.

dación y de la revista de divulgación científica de la SMCTG, nombrando como editor en jefe al Dr. Carlos A Olivares, quien anunció el siguiente congreso, el VII, en la Ciudad de Monterrey, Nuevo León, en septiembre de 2018.

En el VII Congreso celebrado en el Doctors Hospital de la ciudad de Monterrey, Nuevo León, con la participación de los miembros se dieron a conocer los avances científicos de la especialidad: se consolidó la revista con la casa editorial Graphimedic, la cual lleva el nombre oficial de Revista Mexicana de Cirugía Torácica General, y se anunció que el VIII Congreso se llevaría a cabo de nuevo en el Museo de la Vid y el Vino del Valle de Guadalupe la primera semana de octubre de 2019. 
Esto es todo lo que puede hacer la SMCTG por la cirugía de tórax y los cirujanos del país; el sustento para fomentar la educación continua entre los cirujanos de tórax de México es académico, pero idónde queda lo normativo, lo legal o jurídico?, ¿la SMCTG puede con estas labores? La respuesta es no; por eso la necesidad de formar el colegio de cirujanos: hay que impulsar también la creación de más plazas de trabajo y de más lugares de residencia en México.

Para ello el cirujano de tórax necesita ser innovador en su lugar de trabajo y su país, entendiendo por

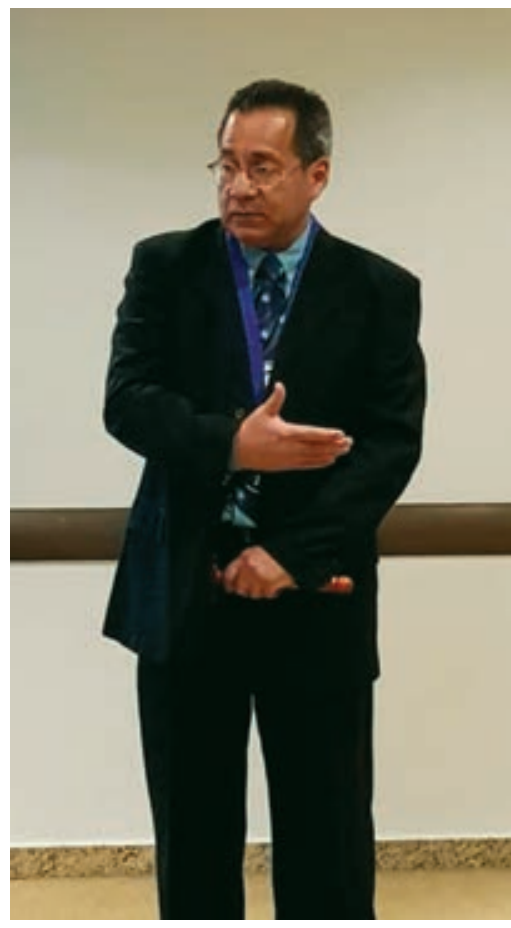

Figura 10:

Toma de protesta del Dr. José Antonio Aburto Salomón. innovación algo diferente que tenga impacto; $;^{1,2}$ un cirujano de tórax lo hace a diario, repercute en la vida de los pacientes en el consultorio, en el quirófano y en la sala de endoscopia. Nuestra labor es única, ningún otro especialista la realiza como el cirujano de tórax.

Debemos, además, progresar y crear nuestro colegio para tener injerencia en la ordenación del ejercicio de la profesión médica, representando a nuestros agremiados y defender los intereses de los mismos; ${ }^{3}$ si nosotros no lo hacemos, nadie lo va a hacer. Debemos hacer política pública y que esas decisiones trasciendan y beneficien a nuestros pacientes, y por qué no, a los mismos cirujanos, con mejores condiciones de trabajo y aranceles.

Participar con órganos públicos y privados en la redacción de normas, proyectos y asuntos inherentes a la cirugía de tórax, además de la elaboración de planes de estudio para la formación de más cirujanos de tórax, con más sedes de nuestra especialidad con un plan único que abarque todo lo largo y ancho del país, garantizando de esta manera a los ciudadanos que el ejercicio de nuestra especialidad se realiza dentro de los requisitos mínimos de calidad y ética como lo describe nuestra Carta Magna en el artículo $5^{0} ;{ }^{4}$ hacerlo valer mediante el Colegio de Cirujanos Torácicos, que es el único y legitimo representante de nuestro gremio. Siempre apoyados en la normatividad que rige nuestro Consejo de Especialidad, que es el Consejo Nacional de Cirugía del Tórax, ${ }^{5}$ motivo por el cual para ser miembro de la SMCTG y fundador del colegio debe tener dicha certificación vigente.

Como dice Richard Branson, "haz que te importen las cosas de manera que puedas hacer algo para resolverlas"; el cirujano de tórax a diario funge como innovador por sus pacientes, por su especialidad, con tecnologías y

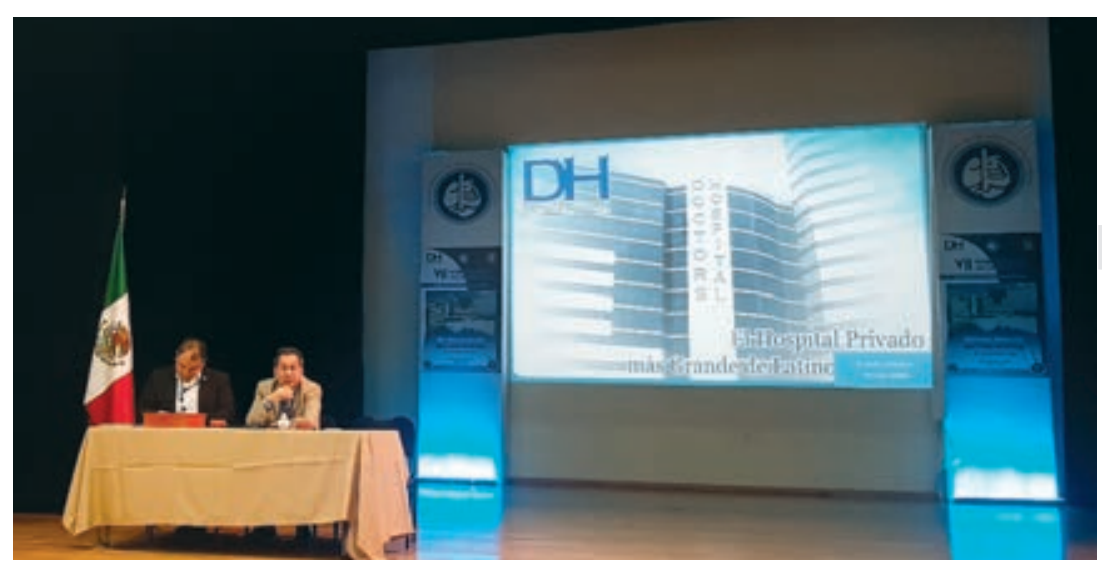

Figura 11:

VII Congreso, Monterrey, Nuevo León, septiembre de 2019. 
procesos nuevos, aplicando lo aprendido cada día; uno está hecho de esa manera, ya lo tenemos programado en nuestro ADN. ${ }^{6}$ El tiempo y la vida útil de un cirujano de tórax son limitados, la vista se va perdiendo y las habilidades se deterioran; sin embargo, hay que tomar en cuenta que nuestra especialidad es de las más completas, pero con un tiempo finito, no podemos operar toda la vida; ${ }^{7} y$ a través de la SMCTG y, en un futuro no muy lejano, del colegio, todos en forma colegiada e individual podremos hacer mejor y más grande nuestra especialidad.

\section{AGRADECIMIENTOS}

A los miembros de la Sociedad Mexicana de Cirujanos Torácicos Generales (SMCTG).

\section{REFERENCIAS}

1. Allen MS. Innovation for life. An Thorac Surg. 2016; 102: 147-125.

2. IBM. Capitalizing on complexity, insights from the 2010 global CEO study. Available in: http: www-0935.ibm.com/services/us/ceo/ ceostudy2010

3. Moreno-Alatorre CR. Los Colegios Médicos en México. Rev Mex Anest. 2007; 30(2): 57-60.

4. Legislación en materia de profesiones. Secretaría de Educación Pública. Gramo Cía. Impresora, S.A. de C.V. 1991.

5. Consejo Nacional de Cirugía del Tórax, A.C. Documentos Normativos, 2018, pp. 1-84.

6. Dyer J, Gregerson $\mathrm{H}$, Christensen $\mathrm{CM}$. The innovators DNA, mastering the skills of disruptive innovators. Boston, Harvard Business School, Publishing, 2011.

7. Careaga-Reyna G. La cirugía cardiotorácica en perspectiva ¿̇un análisis que requieren todas las especialidades? Cir Ciruj. 2011; 79: 100-104.

Conflicto de intereses: Ninguno. 